\title{
Nrf2 Antioxidative System is Involved in Cytochrome P450 Gene Expression and Activity: A Delay in Pentobarbital Metabolism in Nrf2-Deficient Mice
}

\author{
Takashi Ashino, Masayuki Yamamoto, and Satoshi Numazawa
}

Division of Toxicology, Department of Pharmacology, Toxicology and Therapeutics, Showa University School of Pharmacy, Tokyo, Japan (T.A., S.N.); Pharmacological Research Center, Showa University, Tokyo, Japan (T.A., S.N.); and Department of Medical

Biochemistry, Tohoku University Graduate School of Medicine, Sendai, Japan (M.Y.)

accepted April 30, 2020

\section{ABSTRACT}

NF-E2-related factor 2 (Nrf2) is a transcriptional regulator of biologic defense proteins, such as antioxidant proteins and phase II detoxification enzymes. Cytochrome P450 (P450) enzymes have been shown to regulate phase I metabolism of various drugs and are partially regulated by Nrf2; however, the influence of Nrf2 on drug pharmacokinetics is not known. Here, we showed that Nrf2 depletion prolonged the effect of pentobarbital, a sleep-promoting drug. Pretreatment with phenobarbital, a P450 inducer, shortens the sleeping time associated with pentobarbital-induced sedation in wild-type (WT) mice; however, this effect was not observed in $\mathrm{Nrf2}^{-1-}$ mice. Furthermore, the blood pentobarbital concentration was higher in Nrf2 ${ }^{-/-}$mice than in WT mice at 30-60 minutes, and the phenobarbital-induced enhancement of its clearance was attenuated in $\mathrm{Nrf2}^{-1-}$ mice compared with WT mice. Total P450 content was decreased in $\mathrm{Nrf2}^{-1-}$ mouse livers, and the phenobarbitalinduced increase in P450 content was lower in $\mathrm{Nrf2}^{-1-}$ mice than WT mice. Cyp1a2, Cyp2a5, Cyp2c29, and Cyp2e1 gene expression levels under physiologic conditions and Cyp1a2, Сyp2a5, and Сyp2b10 gene expression levels under phenobarbital-treated conditions were lower in $\mathrm{Nrf2}^{-/-}$mice compared with WT mice. Additionally, pentobarbital metabolism in liver microsomes was attenuated by Nrf2 depletion. Taken together, these findings suggested that Nrf2 influenced pentobarbital pharmacokinetics through the regulation of drug metabolism and P450 gene expression. Thus, Nrf2-mediated regulation of P450 may contribute to the biologic defense against increased reactive oxygen species production.

\section{SIGNIFICANCE STATEMENT}

NF-E2-related factor 2 (Nrf2) plays a critical role in the cellular defense against oxidative stress. Nrf2 ${ }^{-/-}$mice with reduced ability to eliminate reactive oxygen species (ROS) showed a significant delay in emergence from pentobarbital-induced sleep, which was associated with decreased P450 activities and gene expression. Our findings provide that Nrf2 dysfunction or ROS that exceed a threshold level of the eliminating ability of the Nrf2 system may reduce P450 activity.

\section{Introduction}

Cytochrome P450s (P450s) are a large family of heme-containing monooxygenase enzymes that oxidatively metabolize xenobiotics such as drugs and environmental pollutants (Gonzalez, 1988; Isin and Guengerich, 2007). Numerous factors, including xenobiotics (e.g., phenobarbital) and diseases, regulate P450 enzymatic activity (Morgan, 1997; Wang and Negishi, 2003; Aitken et al., 2006; Hakkola et al., 2018). Alterations in the gene expression and activity of P450s can affect the pharmacokinetics of administered drugs, leading to changes in the duration and intensity of their intended efficacies. Hypoactivity of P450 enzymes can result in increased blood levels of the therapeutic drugs, followed by adverse events. Therefore, elucidation of the underlying

This work was supported by Japan Society for the Promotion of Science (JSPS) KAKENHI [18K08115] (to T.A.) and Takeda Science Foundation [2015061858] (to T.A.).

There is no conflict of interest.

https://doi.org/10.1124/dmd.120.000010. regulatory mechanism of $\mathrm{P} 450$ s may improve clinical predictions of the pharmacokinetics and adverse effects of these medicines.

Excess reactive oxygen species (ROS), including superoxide anion and hydrogen peroxide, can induce oxidative damage to various cells, followed by the development and progression of multiple organ dysfunction, including atherosclerosis, diabetes mellitus, and cancer (Roberts and Sindhu, 2009). Conversely, ROS have also been recognized as intracellular signaling molecules and are constitutively produced during various biologic processes, including energy production, immune response, and wound healing (Griffiths et al., 2017). Additionally, the $\mathrm{P} 450$ drug metabolizing system is known to produce ROS in both the presence and absence of substrates (Zangar et al., 2004). Generally, P450-mediated oxidation requires input of two electrons by the flavoprotein NADPH-P450 reductase to activate oxygen and insert an oxygen atom into the substrate molecule (Zangar et al., 2004). ROS accumulation can occur through NADPH consumption by microsomal $\mathrm{P} 450$, which is reported to be involved in nonalcoholic fatty liver disease (Aubert et al., 2011), hypertension (Sahan-Firat et al., 2010), and diabetes (Eid et al., 2009). These reports suggested that P450 activity

ABBREVIATIONS: ARE/StRE, antioxidant- or stress-response elements; CAR, constitutive androstane receptor; HPLC, high-performance liquid chromatography; Nrf2, NF-E2-related factor 2; P450, cytochrome P450; PCR, polymerase chain reaction; ROS, reactive oxygen species; WT, wild type. 
was tightly controlled by regulating its gene expression in ROS-related pathologic conditions to protect against organ injury.

In the context of biologic defense, excess ROS can induce oxidative damage; hence, cellular redox balance is tightly controlled by various antioxidant systems. NF-E2-related factor 2 (Nrf2), a redox-regulated transcription factor, plays a critical role in the cellular defense system by activating gene expression of biologic defense proteins, such as antioxidant proteins, glutathione synthesis-associated proteins, and phase II detoxification enzymes, via its binding to antioxidant- or stress-response elements (ARE/StRE) (Yamamoto et al., 2018). We previously demonstrated that Nrf2 is involved in the expression of Cyp2b10 and Cyp2a5 during the steady state and their induction by phorone, an $\alpha, \beta$-unsaturated carbonyl compound, and phenobarbital, a classic P450 inducer (Ashino et al., 2014). Furthermore, oxidative stress-induced transcription of Cyp2a5 is activated through direct binding of Nrf2 to its ARE/StRE in the 5'-flanking region (Abu-Bakar et al., 2007). However, ARE/StRE have not been identified in other P450 genes.

Several studies have reported that inflammatory diseases (e.g., rheumatoid arthritis and chronic heart failure) and infection are risk factors for decreased P450 gene expression and activity (Morgan, 1997; Mayo et al., 2000; Frye et al., 2002; Ashino et al., 2007). In addition, these diseases increase ROS levels, resulting in oxidative stress. We have previously demonstrated that lipopolysaccharide and Bacillus Calmette-Guérin downregulate the levels of Cyp3a11 and Cyp2c29 in mouse livers (Ashino et al., 2004), as well as induce heme oxygenase-1, a well known Nrf2 target gene (Oguro et al., 2002; Ashino, unpublished data). These observations suggest that $\mathrm{Nrf} 2$ is involved in the expression of various P450 species. However, the influence of Nrf2 on P450 gene expression, except for Cyp2a5 and Cyp2b10, and drug pharmacokinetics has not been demonstrated.

Pentobarbital, an anesthetic barbiturate, is a widely used pharmacological or toxicological study model. In this study, we demonstrate that Nrf 2 depletion prolonged the sleeping time with pentobarbital sedation. Nrf2 depletion maintains high blood levels of pentobarbital, through P450 and ferritin-mediated metabolic suppression, in both physiologic and phenobarbital-induced P450 expression conditions. Collectively, Nrf2 depletion-increased ROS was shown to downregulate P450 gene expression through a negative feedback mechanism, thereby decreasing P450 protein levels and their activities and maintaining blood concentrations of the drug. These findings suggest that Nrf2 activation may be used as an indicator of drug metabolizing capacity in oxidative stress-related diseases.

\section{Materials and Methods}

Materials. Pentobarbital and phenobarbital sodium salts were acquired from Tokyo Chemical Industry (Tokyo, Japan). All other reagents used were of the highest grade commercially available.

Animals and Treatments. All animal experiments were conducted in accordance with the protocols approved by the Institutional Animal Care and Use Committees of Showa University (29015), in accordance with the Standards Relating to the Care and Management of Experimental Animals in Japan. Male C57BL/6 mice (8 weeks old) were purchased from Japan SLC (Shizuoka, Japan). The $\mathrm{Nrf}^{-/-}$mice were established by Itoh et al. (1997). Subsequently, C57BL/6 mice were mated with $\mathrm{Nrf}^{-1-}$ mice to generate wild-type (WT) and deficient mice progenies. Pentobarbital $(50 \mathrm{mg} / \mathrm{kg})$ and phenobarbital $[100 \mathrm{mg} / \mathrm{kg}$; dose used for most P450 induction studies (Ashino et al., 2014)] were dissolved in saline and injected intraperitoneally. Control groups were injected with the same volume of vehicle (saline).

Measurement of Sleeping Time with Pentobarbital Sedation. All experiments were started between 11:00 AM and 2:00 PM. Mice were injected with pentobarbital, and the time to loss of righting reflex was monitored. The awakening time was defined as the time of righting of the animal.
Preparation of Liver Microsomes and Measurement of Total P450 Content. Microsomal fractions from mouse livers were prepared as described previously (Ashino et al., 2011). Total hepatic P450 content was determined from a carbon monoxide difference spectrum of dithionite-treated microsomes suspended in $0.1 \mathrm{M}$ sodium-potassium phosphate buffer $(\mathrm{pH}$ 7.4) and recorded with an MPS-2450 spectrophotometer (Shimadzu, Kyoto, Japan) using a difference extinction coefficient $(450-490 \mathrm{~nm})$ of $91 \mathrm{mM}^{-1} \mathrm{~cm}^{-1}$ as described by Omura and Sato (1964). Microsomal protein concentration was determined using the BCA protein assay.

Measurement of Blood Pentobarbital Concentrations. Whole blood samples were collected from different mice under anesthesia by injecting medetomidine $(0.3 \mathrm{mg} / \mathrm{kg})$, midazolam $(4 \mathrm{mg} / \mathrm{kg})$, and butorphanol $(5 \mathrm{mg} / \mathrm{kg})$ intraperitoneally because pentobarbital shows no analgesic effect. Plasma samples were prepared from whole blood. Plasma pentobarbital concentrations were determined by using a high-performance liquid chromatography (HPLC)-UV assay based on previous reports (Kelner and Bailey, 1983). Briefly, hexobarbital (as an internal standard; $10 \mu \mathrm{l} ; 0.1 \mu \mathrm{g} / \mu \mathrm{l}$ in acetonitrile) and acetonitrile $(190 \mu \mathrm{l})$ were added to $100 \mu \mathrm{l}$ of plasma, and the mixture was vortexed for 30 second and centrifuged at 20,000 $\mathrm{g}$ for 10 minutes to separate the precipitated proteins. Clear supernatant $(50 \mu \mathrm{l})$ was injected into the HPLC system. The HPLC system consisted of an LC-10AD liquid chromatograph pump (Shimadzu), an SPD-10A UV-VIS detector (Shimadzu), an SIL-10A auto injector (Shimadzu), a CMB-10A communications bus module (Shimadzu), and a $4.6 \times 150 \mathrm{~mm}$ Luna $5 \mu \mathrm{m} \mathrm{C18}(2)$ column (Shimadzu GLC, Tokyo, Japan). The mobile phase consisted of methanol, $0.16 \mathrm{M}$ sodium-potassium phosphate buffer ( $\mathrm{pH} 6.6$ ), and tetrahydrofuran (50:43: 7) at a flow rate of $1 \mathrm{ml} / \mathrm{min}$. The eluate was monitored at $220 \mathrm{~nm}$. Under the present chromatographic conditions, hexobarbital and pentobarbital were eluted at 5 and 6 minutes, respectively. Pentobarbital was quantified by comparison with the standard curves $(6.25-100 \mu \mathrm{g} / \mathrm{ml})$ using the area ratio method.

Pentobarbital Metabolic Activity in the Hepatic Microsomes. Pentobarbital metabolic activity assay was performed in a reaction mixture $(500 \mu l)$ containing hepatic microsomes (500 $\mu \mathrm{g}$ protein), $5 \mathrm{mM} \mathrm{MgCl}_{2}$, pentobarbital (50 $\mu \mathrm{M}$ in Fig. 3 or $6.25-100 \mu \mathrm{M}$ in Fig. 4), and the NADPH-generating system (5 mM glucose-6-phosphate, $0.6 \mathrm{mM} \beta$-nicotinamide adenine dinucleotide phosphate ${ }^{+}$, and $1 \mathrm{U}$ glucose-6-phosphate dehydrogenase) in $100 \mathrm{mM}$ phosphate

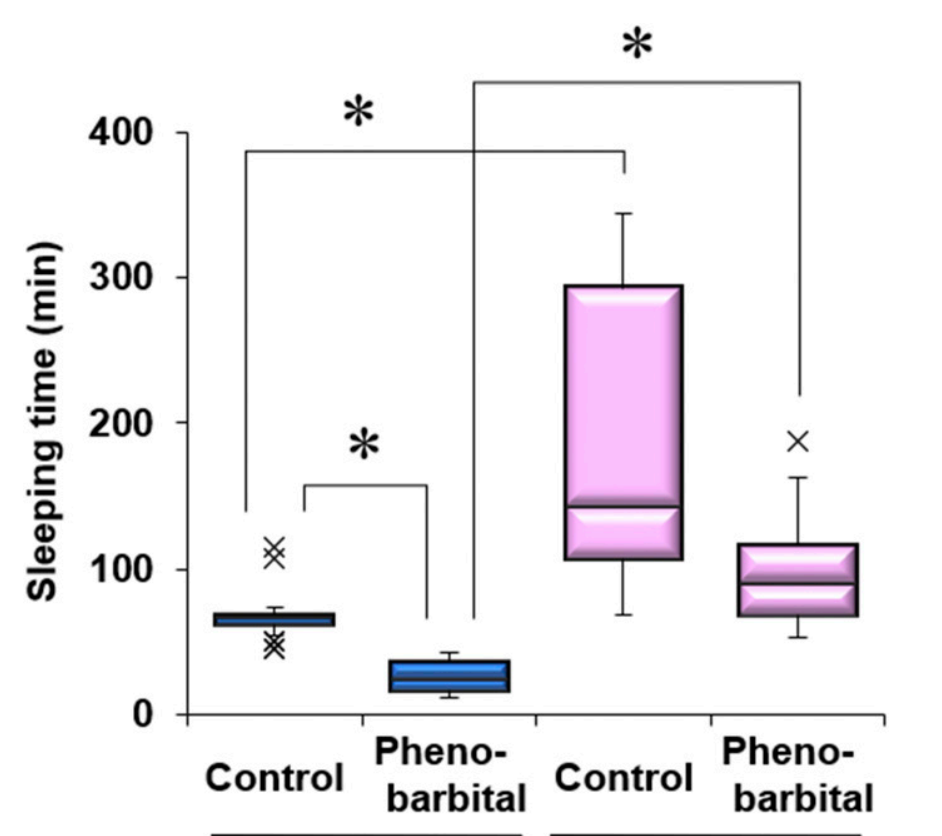

\section{WT $\mathrm{Nrf2}^{-1-}$}

Fig. 1. Pentobarbital-induced sleeping time in WT and $\mathrm{Nrf2} 2^{-1-}$ mice treated with or without phenobarbital. Mice were pretreated with phenobarbital $(100 \mathrm{mg} / \mathrm{kg}$, i.p.) or saline. After 24 hours, the mice were injected with pentobarbital $(50 \mathrm{mg} / \mathrm{kg}$, i.p. $)$, and sleeping time was monitored $(n=15, * P<0.05)$. Horizontal lines and boxes indicate medians with interquartile range. Whiskers indicate \pm 1.5 interquartile range, and points beyond whiskers indicate outliers. 
buffer ( $\mathrm{pH}$ 7.4). The reaction mixture was incubated at $37^{\circ} \mathrm{C}$ for 15 minutes, and the reaction was stopped by adding ice-cold $490 \mu l$ acetonitrile. Hexobarbital (10 $\mu \mathrm{l} ; 1 \mathrm{mM}$ in acetonitrile) was added to the reaction mixture as an internal standard, and the mixture was vortexed for 30 second and centrifuged at $1800 \mathrm{~g}$ for 10 minutes to separate the precipitated proteins. Clear supernatant $(50 \mu \mathrm{l})$ was injected into the HPLC system. Pentobarbital was quantified as mentioned above by comparing with the standard curves $(3.125-100 \mathrm{nmol} /$ reaction mixture). Pentobarbital metabolic activity was calculated from the rate of decrease. Controls without microsomes showed no decrease in pentobarbital.

Quantitative Real-Time Polymerase Chain Reaction. Total RNA was isolated from the livers using the PureLink RNA Mini Kit (Invitrogen), and first-

A

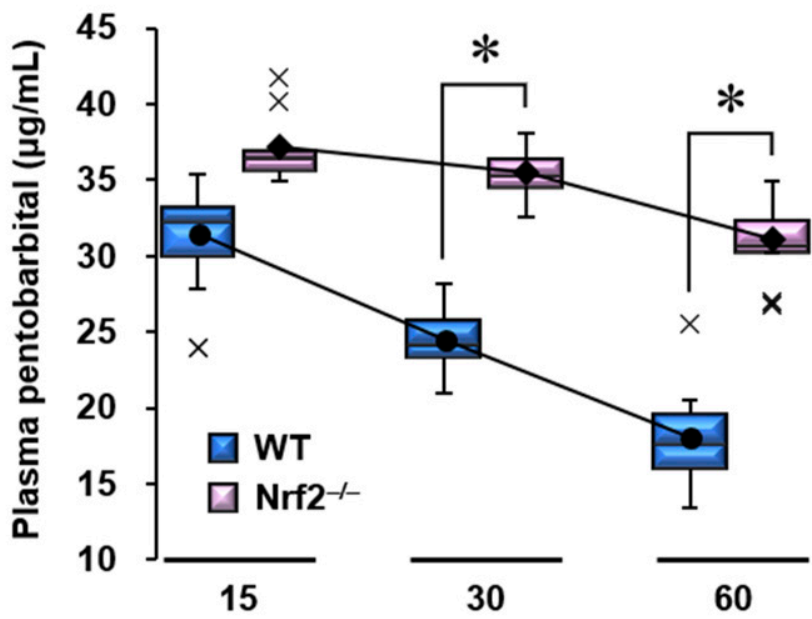

Time after pentobarbital injection (min)

B

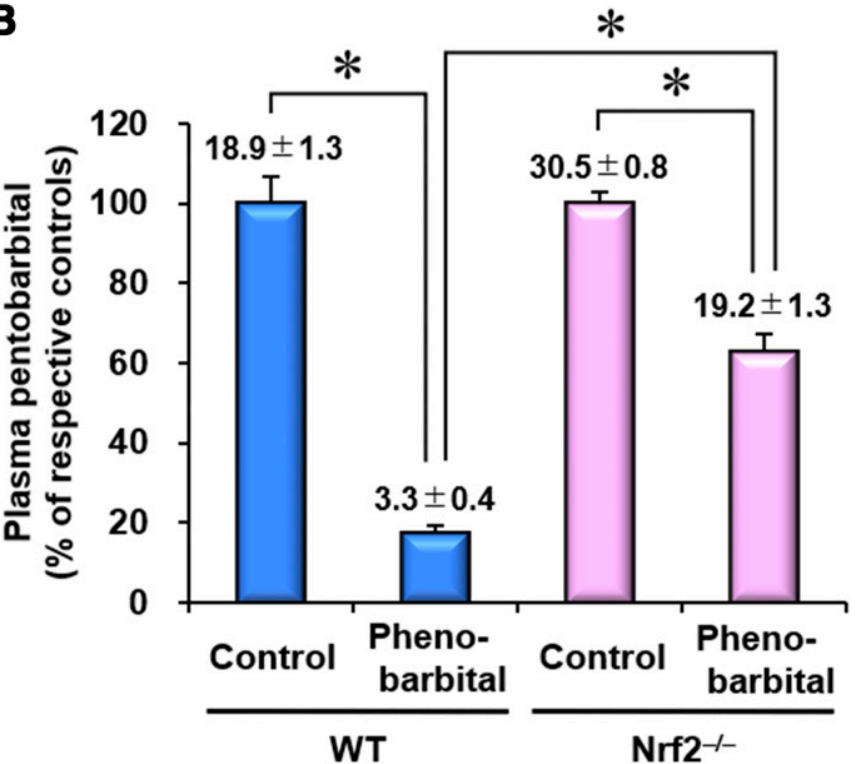

Fig. 2. Rate of decline in blood pentobarbital concentrations in WT and $\mathrm{Nrf} 2^{-/-}$ mice treated with or without phenobarbital. (A) Mice were injected with pentobarbital (50 mg/kg, i.p.), and their plasma samples were prepared from whole blood at the indicated time points. Line graphs represent averaged data expressed as plasma pentobarbital concentrations. Horizontal lines and boxes indicate medians with interquartile range. Whiskers indicate \pm 1.5 interquartile range, and points beyond whiskers indicate outliers $(n=10, * P<0.05)$. (B) Mice were pretreated with phenobarbital $(100 \mathrm{mg} / \mathrm{kg}$, i.p.) or saline. After 24 hours, the mice were injected with pentobarbital $(50 \mathrm{mg} / \mathrm{kg}$, i.p.), and their plasma samples were prepared at 60 minutes postpentobarbital. Bar graphs represent averaged pentobarbital concentrations expressed as the percentage of the respective controls. Numerals indicate plasma pentobarbital concentrations (micrograms per milliliter). Values represent means \pm S.E.M. $\left(n=11,{ }^{*} P<0.05\right)$.

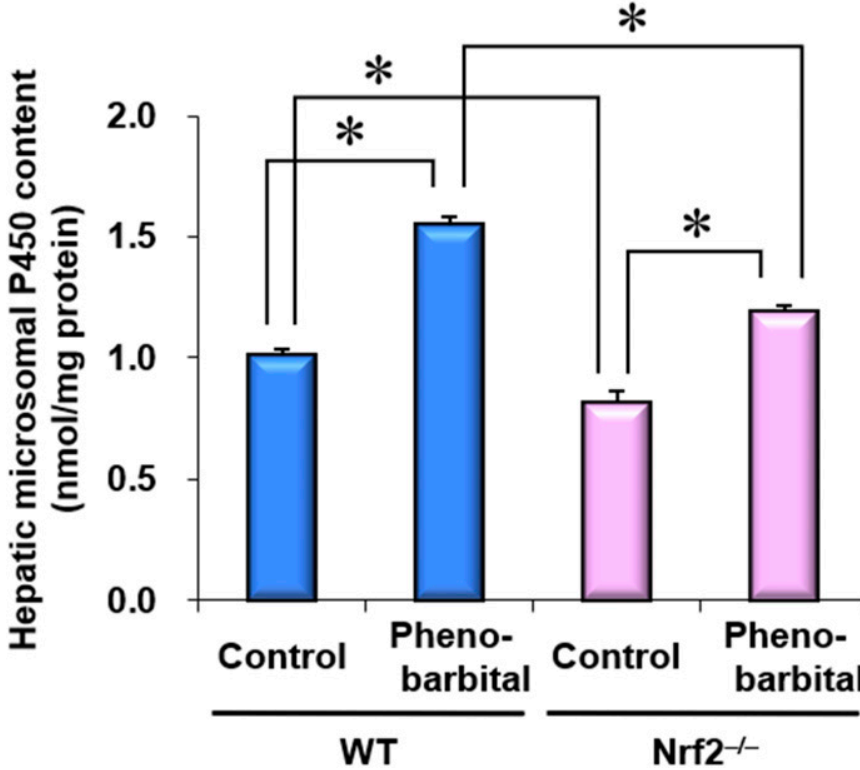

Fig. 3. Microsomal P450 content in liver microsomes of WT and $\mathrm{Nrf} 2^{-1-}$ mice treated with or without phenobarbital. Mice were injected with phenobarbital $(100 \mathrm{mg} / \mathrm{kg}$, i.p.) or saline, and the livers were excised 24 hours after treatment. Total P450 content in the liver microsomal proteins was assayed from the carbon monoxide difference spectrum of the reduced protein. Values represent means \pm S.E.M. $\left(n=4-6,{ }^{*} P<0.05\right)$.

stranded cDNA was synthesized using the PrimeScript RT Master Mix (Takara Bio, Shiga, Japan). Duplex TaqMan real-time polymerase chain reaction (PCR) was performed using the TaqMan Fast Advanced Master Mix (Applied Biosystems), a FAM-labeled probe for the target mRNA, and a VIC-labeled probe for the housekeeping gene ( $\beta$-actin) according to the manufacturer's protocol on a StepOne real-time PCR system (Applied Biosystems). The mRNA levels were measured relative to $\beta$-actin mRNA expression in each run. All predesigned PCR primers and TaqMan MGB probes were purchased from Applied Biosystems.

Statistical Analysis. Values are expressed as medians with interquartile \pm 1.5 interquartile range (Fig. 1; Fig. 2A) or means \pm S.E.M. (Fig. 2B; Figs. 3 and 5-7). The values beyond \pm 1.5 interquartile range were defined as the outliers. Time courses were compared among groups by Kruskal-Wallis test and post hoc Steel's test. Two group means were compared by Mann-Whitney's $U$ test, and three or more group means by Kruskal-Wallis test, followed by post hoc Scheffe's tests. Statistical significance was accepted at $P<0.05$.

\section{Results}

Loss of Nrf2 Prolongs Pentobarbital-Induced Sleeping Time Without Effects on Phenobarbital-Induced Sleeping Time. Alteration of P450 enzymatic activity can affect pharmacokinetics of drugs. We previously demonstrated that Nrf2 is involved in expression of some P450 genes using $\mathrm{Nrf}^{-1-}$ mice (Ashino et al., 2014). Thus, we investigated the changes in drug-metabolizing activity in $\mathrm{Nrf}^{-/-}$and age-, sex-, and body weight-matched WT mice following pentobarbital (a known substrate for P450s) administration (Fig. 1). The dose of pentobarbital $(50 \mathrm{mg} / \mathrm{kg})$ used was that at which the WT mice recovered in approximately 60 minutes after the loss of righting reflex. Compared with WT mice, $\mathrm{Nrf}^{-1-}$ mice showed a significant delay in emergence from pentobarbital-induced sleep (average: WT, 69 minutes vs. $\mathrm{Nrf} 2^{-l-}, 192$ minutes). We also reported that $\mathrm{Nrf} 2^{-1-}$ mice showed attenuated Cyp2b10 and Cyp2a5 induction by phenobarbital, a classic P450 inducer (Ashino et al., 2014). In WT mice, pretreatment with phenobarbital for 24 hours shortened pentobarbital-induced sleeping time, but this effect was not observed in $\mathrm{Nrf} 2^{-/-}$mice. 

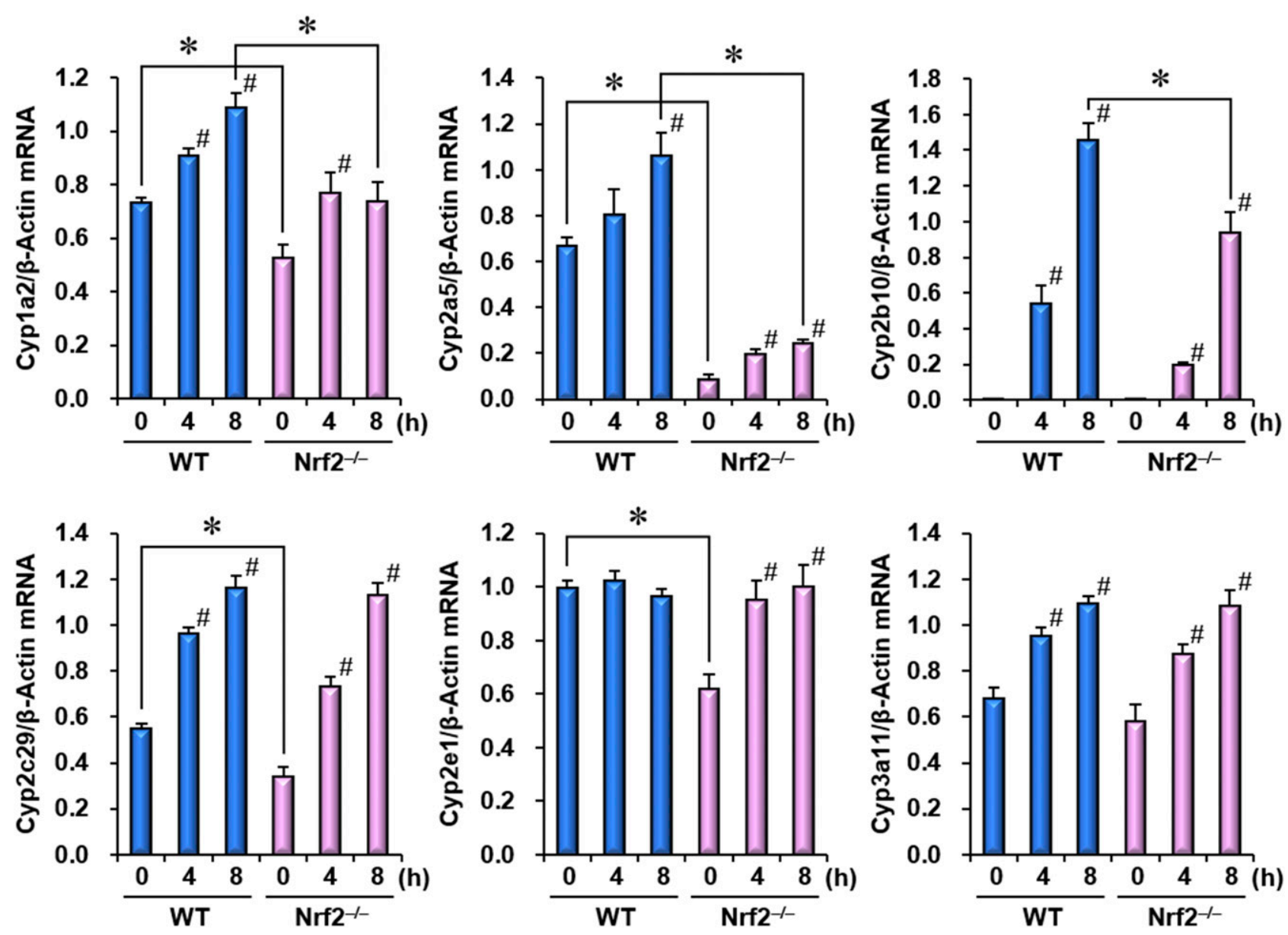

\section{Time after phenobarbital injection}

Fig. 4. Gene expression of various P450 molecular species in livers of WT and $\mathrm{Nrf}^{-1-}$ mice treated with or without phenobarbital. Mice were injected with phenobarbital $(100 \mathrm{mg} / \mathrm{kg}$, i.p.) or saline, and the livers were excised at the indicated times. Various P450 mRNA levels were determined by real-time PCR and semiquantified by normalizing to $\beta$-actin mRNA expression. Values represent means \pm S.E.M. $(n=5) .{ }^{\#} P<0.05$ vs. respective controls $(0$ hour $) .{ }^{*} P<0.05$ vs. WT.

Clearance of Blood Pentobarbital Is Delayed in $\mathrm{Nrf}^{-1-}$ Mice Both with and Without Phenobarbital Pretreatment. To determine whether Nrf2 deficiency influences pentobarbital pharmacokinetics, we examined time-dependent changes in blood concentrations of pentobarbital in WT and Nrf2 ${ }^{-1-}$ mice. As shown in Fig. 2A, 15 minutes after treatment, there was no significant difference in pentobarbital concentrations between WT and $\mathrm{Nrf} 2^{-1-}$ mice. At 30 minutes, pentobarbital blood levels were higher in $\mathrm{Nrf} 2^{-/-}$mice than WT mice; the difference was even more pronounced at 60 minutes, indicating a delay in pentobarbital metabolism in $\mathrm{Nrf} 2^{-1-}$ mice. Furthermore, we examined the effect of phenobarbital on the pentobarbital metabolic rate in WT and $\mathrm{Nrf} 2^{-1-}$ mice (Fig. 2B). Phenobarbital pretreatment for 24 hours markedly decreased blood pentobarbital levels in WT mice, whereas this effect was attenuated in $\mathrm{Nrf} 2^{-1-}$ mice.

Nrf2 Depletion Decreases Hepatic Microsomal Cytochrome P450 Content. Since Nrf2 depletion resulted in sustained high blood levels of pentobarbital, which is metabolized by P450 enzymes, we examined changes in hepatic P450 content. As shown in Fig. 3, physiologic and phenobarbital-induced hepatic P450 levels in $\mathrm{Nrf}^{-/-}$ mice were significantly lower than WT mice.

Nrf2 Depletion Influences P450 Expression Levels in the Liver under Physiologic and Inducing Conditions. To determine which P450 enzymes were influenced by Nrf2 depletion, we examined the expression levels of constitutive-type P450 (Fig. 4). Under physiologic conditions, Nrf2 $2^{-/-}$mice showed low levels of Cyp1a2, Cyp2a5, Cyp2c29, and Cyp2e1 expression compared with WT mice. Under phenobarbital-inducing conditions, although almost all $\mathrm{P} 450$ species were increased in both WT and Nrf $2^{-1-}$ mice, Cyp1a2, Cyp2a5, and Cyp2b10 expression levels were lower in $\mathrm{Nrf}^{-1-}$ mice than WT mice. Expression of the Cyp3a11 gene did not show significant change between WT and Nrf2 $2^{-l-}$ mice before and after phenobarbital treatment.

Nrf2 Depletion Downregulates Ferritin Gene Expression. P450 enzymes are capable of performing catalysis through reduction or oxidation of their heme iron. Thus, the supply of heme to the apoprotein of P450 enzymes is important for their catalytic activity. Ferritin, an intracellular iron storage protein composed of ferritin heavy $(\mathrm{H})$ and light (L) chains, has been postulated to act as an intermediate for heme synthesis (Richardson and Ponka, 1997). As shown in Fig. 5, under physiologic conditions, gene expression levels of both ferritin heavy $(\mathrm{H})$ and light (L) chains were lower in $\mathrm{Nrf}^{-1-}$ mouse livers than WT. Furthermore, phenobarbital induced expression of both ferritin heavy (H) and light (L) chains. However, expression levels of ferritin heavy $(\mathrm{H})$ chain, and not ferritin light (L) chain, was lower in $\mathrm{Nrf}^{-/-}$mice than WT mice, even in phenobarbital induced conditions.

Pentobarbital Metabolic Activity Is Decreased in Nrf2 ${ }^{-/-}$Mouse Livers. Nrf2 depletion decreased the levels of several P450 molecular species and ferritin gene expression. We therefore examined pentobarbital metabolic activity in liver microsomes of phenobarbital-treated and 


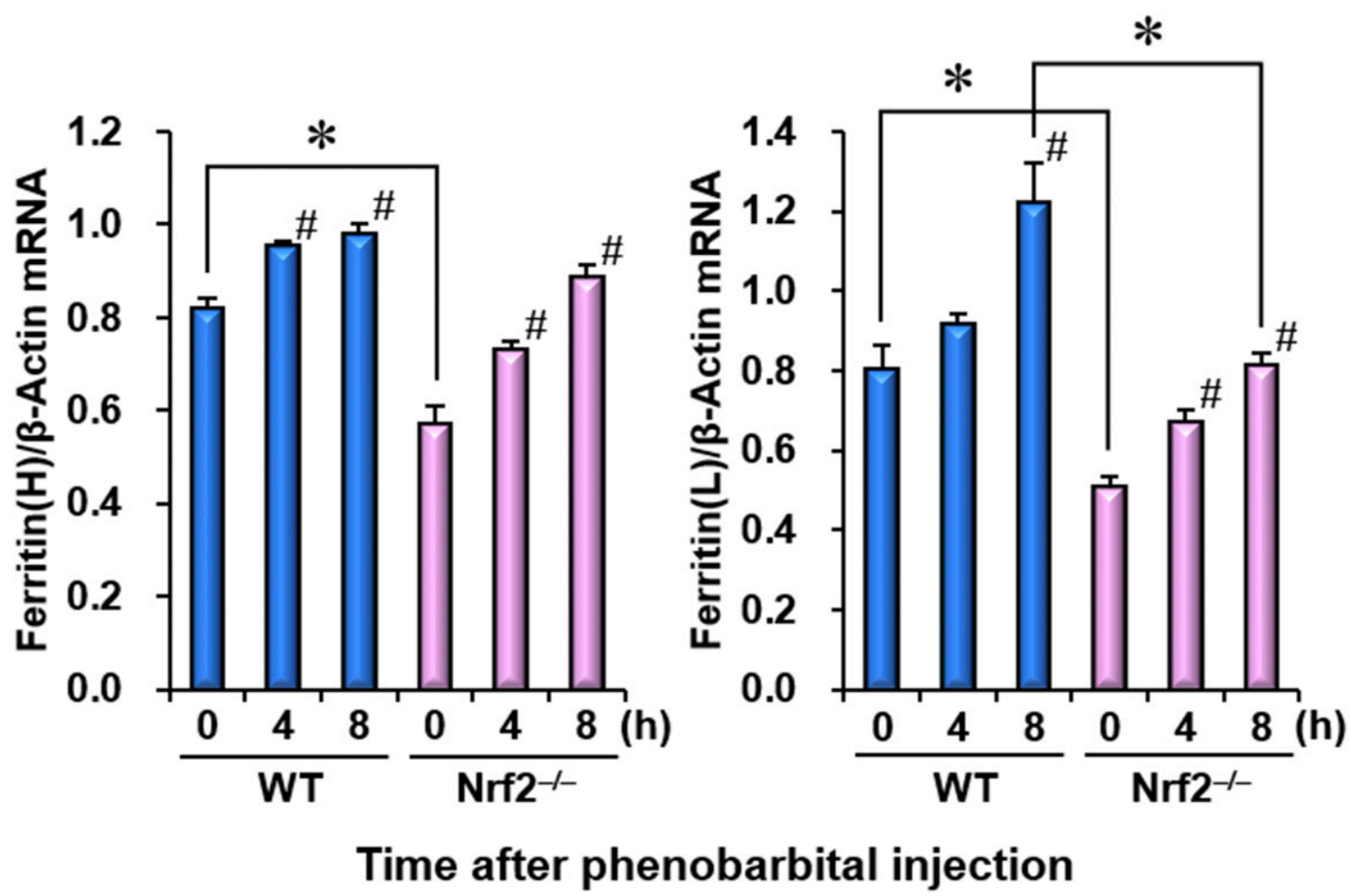

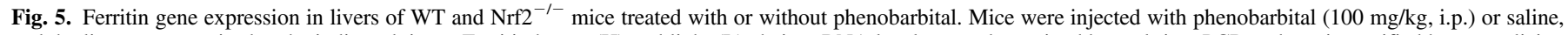

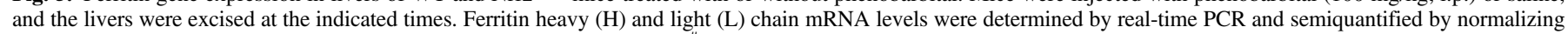
to $\beta$-actin mRNA expression. Values represent means \pm S.E.M. $(n=5)$. ${ }^{\#} P<0.05$ vs. respective controls $(0$ hour $)$. ${ }^{*} P<0.05$ vs. WT.

untreated WT and Nrf2 $2^{-\prime-}$ mice (Fig. 6). Consistent with sleeping time and blood pentobarbital levels, pentobarbital metabolic activity in uninduced liver microsomes was significantly lower in $\mathrm{Nrf} 2^{-1-}$ mice compared with WT mice. Furthermore, phenobarbital enhanced pentobarbital metabolic activity in both $\mathrm{WT}$ and $\mathrm{Nrf} 2^{-1-}$ mouse liver microsomes at 24 hours after treatment. However, pentobarbital metabolic activity in phenobarbital-treated $\mathrm{Nrf}^{-1-}$ mouse liver microsomes was significantly lower than WT microsomes. To characterize the mode of inhibition of pentobarbital metabolic activity in $\mathrm{Nrf}_{2}^{-1-}$ mice, we performed kinetic analysis (Fig. 7). Michaelis-Menten kinetics (Fig. 7A) and Lineweaver-Burk plots (Fig. 7B) showed that pentobarbital metabolic activity was noncompetitively suppressed in $\mathrm{Nrf} 2^{-/-}$ mouse livers, with $K_{\mathrm{m}}$ values of approximately $25 \mu \mathrm{M}$ (Fig. 7C), suggesting that Nrf2 depletion did not affect the affinity of P450 enzymes to pentobarbital.

\section{Discussion}

In clinical reports, patients under oxidative stress conditions have been shown to suppress $\mathrm{P} 450$ metabolizing activities, including mephenytoin (a substrate for CYP2C19) and caffeine (a substrate for CYP1A2) in patients with congestive heart failure (Frye et al., 2002). Furthermore, patients after surgical operations have been shown to suppress erythromycin metabolic capacity (a substrate for CYP3A4) (Haas et al., 2003). Other reports have implicated ROS in the transcriptional regulation of P450s (Barouki and Morel, 2001), suggesting that ROS-induced oxidative stress may have a role in suppressing drug metabolizing activity. At present, the role of oxidative stress defensive transcription factor Nrf2 on drug pharmacokinetics is unclear. This study revealed a novel role for Nrf2 in which it modulates drug pharmacokinetics by regulating P450 expression. In WT mice, Nrf2-activated antioxidant proteins eliminated ROS, thereby maintaining
P450 expression and activity (Fig. 8, upper). In contrast, $\mathrm{Nrf}^{-1-}$ mice had a reduced ability to eliminate ROS, resulting in elevated ROS levels that suppressed $\mathrm{P} 450$ expression and activity through a negative feedback mechanism (Fig. 8, lower). Thus, our results suggest that

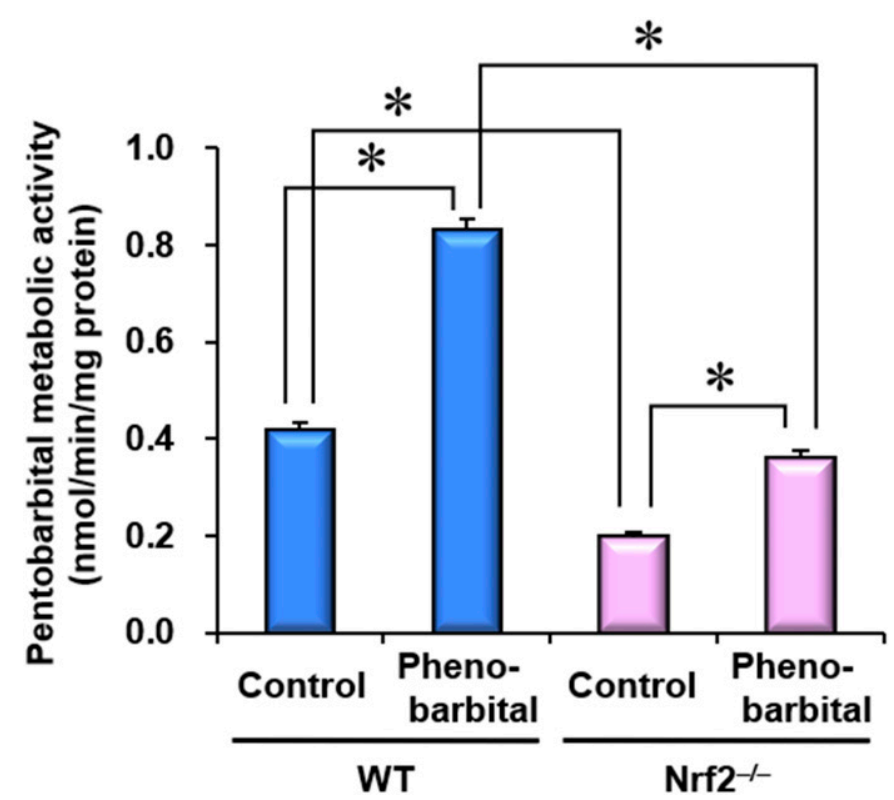

Fig. 6. Pentobarbital metabolic activity in liver microsomes from $\mathrm{WT}$ and $\mathrm{Nrf} 2^{-1-}$ mice treated with or without phenobarbital. Mice were injected with phenobarbital (100 mg/kg, i.p.) or saline, and the livers were excised 24 hours after treatment. Pentobarbital metabolic activity was determined from the rate of decrease in pentobarbital by incubation with the liver microsomes. Values represent means \pm S.E.M. $\left(n=4-6,{ }^{*} P<0.05\right)$. 
A

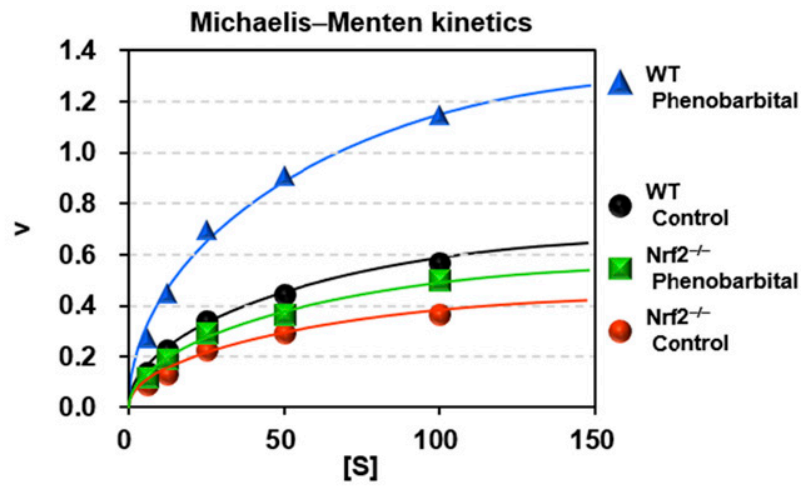

B

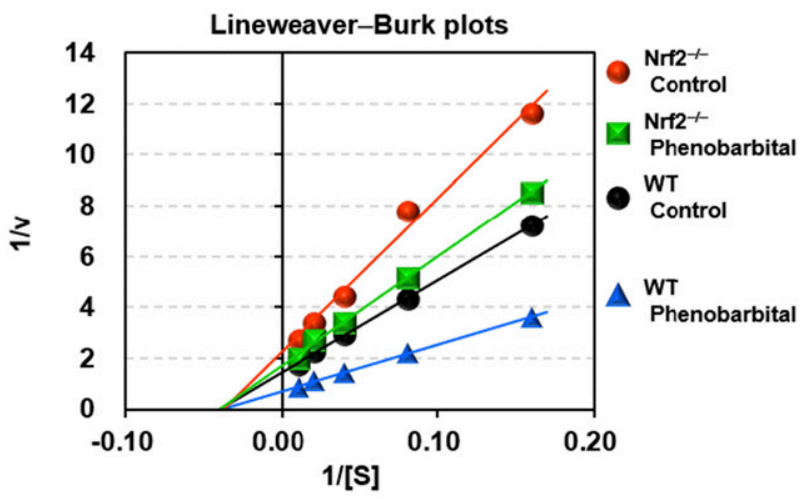

\begin{tabular}{|c|c|c|c|}
\hline & $\begin{array}{l}\text { Vmax } \\
\text { (nmol/min/mg protein) }\end{array}$ & $\underset{(\mu \mathrm{M})}{\mathrm{Km}}$ \\
\hline \multirow{2}{*}{ WT } & Control & $0.68(100 \%)$ & 24.8 \\
\hline & Phenobarbital & $1.40(204 \%)$ & 25.6 \\
\hline \multirow{2}{*}{$\mathrm{Nrf2}^{-1-}$} & Control & $0.44(100 \%)$ & 26.6 \\
\hline & Phenobarbital & $0.58(132 \%)$ & 24.8 \\
\hline
\end{tabular}

Fig. 7. Kinetic analysis of pentobarbital metabolic activity of liver microsomes from WT and Nrf2 $2^{-1-}$ mice treated with or without phenobarbital. (A and B) Michaelis-Menten kinetics (A) and Lineweaver-Burk plots (B) of pentobarbital metabolic activity in the liver microsomes of WT and $\mathrm{Nrf}^{-1-}$ mice at 24 hours after phenobarbital treatment $(100 \mathrm{mg} / \mathrm{kg}$, i.p.). Microsomal protein was isolated from three different mouse livers and combined. [S] and $\mathrm{v}$ indicate pentobarbital concentration (micromolars) and pentobarbital metabolic activity (nmol/min/mg protein), respectively. (C) The $V_{\max }$ and $K_{\mathrm{m}}$ values were calculated from Lineweaver-Burk plots.

Nrf2 modulates the duration and intensity of drug effects to maintain redox balance.

The length of pentobarbital-induced sleeping time is inversely related to the rate of drug metabolism, and its duration of action can be altered by induction or inhibition of P450s (Sirisangtragul and Sripanidkulchai, 2013; Kovacs et al., 2018). In this study, Nrf2 depletion prolonged sleeping time by sustaining high blood levels of pentobarbital under physiologic conditions. These results indicate that pentobarbital metabolism is delayed by $\mathrm{Nrf} 2$ depletion, as evidenced by suppressed pentobarbital metabolism in the liver microsomes of $\mathrm{Nrf} 2^{-1-}$ mice. Barbiturates are metabolized by several $\mathrm{P} 450$ species, including CYP2B6 and CYP3A4 in humans (Tsuji et al., 1996; Sirisangtragul and Sripanidkulchai, 2013). Since P450 enzymes show broad substrate specificities, other $\mathrm{P} 450$ species may contribute to pentobarbital metabolism. In this study, Nrf2 depletion decreased total P450 content and downregulated gene expression of several $\mathrm{P} 450$ species, such as Cyp1a2, Cyp2a5, Cyp2c29, and Cyp2e1, under physiologic conditions.
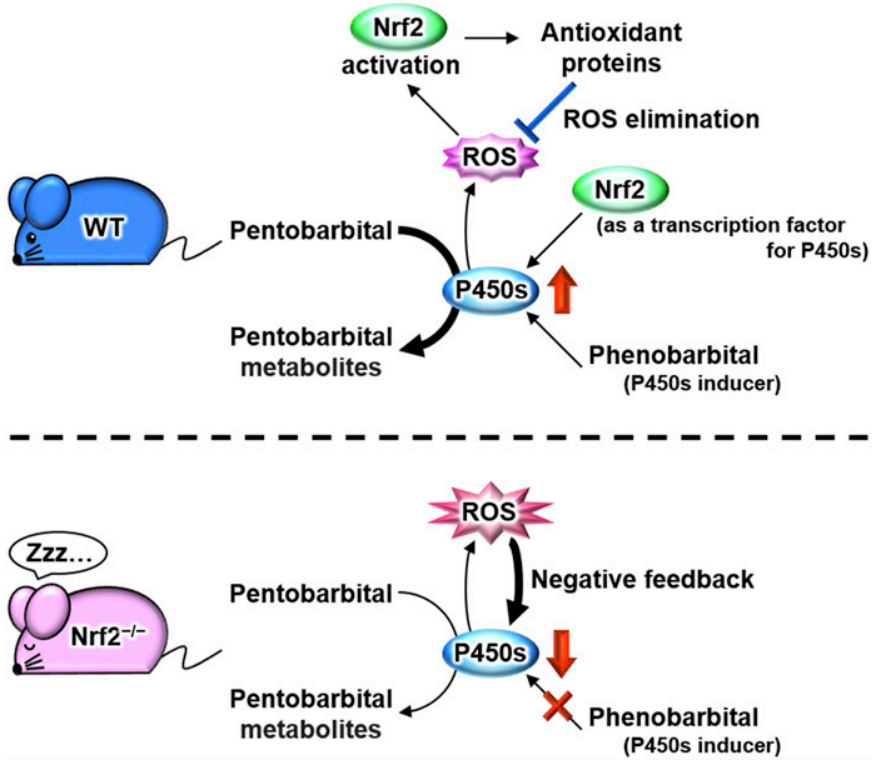

Fig. 8. Proposed model for the decline in $\mathrm{P} 450$ gene expression and activity by $\mathrm{Nrf} 2$ depletion. Increase in ROS downregulates $\mathrm{P} 450$ gene expression through a negative feedback mechanism, thereby decreasing P450 protein expression and activities in $\mathrm{Nrf}^{-1-}$ mice, which have suppressed ROS elimination ability due to loss of antioxidant function, to maintain drug concentrations in the blood.

Consistent with our observations, portal vein ligation of the liver transiently elevated pentobarbital-induced sleeping time, which was associated with decreased $\mathrm{P} 450$ activities and gene expression of CYP1A, CYP2B, and CYP2C in rats (Kovacs et al., 2018). Collectively, the sustained efficacy of pentobarbital by Nrf2 depletion is attributed to P450 downregulation.

This study also evaluated changes in pentobarbital pharmacokinetics using the P450 inducer phenobarbital in Nrf2 $2^{-1-}$ mice. Phenobarbital shortened pentobarbital-induced sleeping time. This was abolished by Nrf2 depletion. Consistent with sleeping time, phenobarbital-enhanced pentobarbital metabolic activity was suppressed by $\mathrm{Nrf} 2$ depletion both in vivo and in vitro. Phenobarbital is a classic inducer of the drug metabolizing P450 genes, especially the CYP2B subfamily. In this study, phenobarbital induced expression of all P450 genes, except for Cyp2e1 in WT mice. Cyp1a2, Cyp2a5, and Cyp2b10 gene expression levels in $\mathrm{Nrf} 2^{-/-}$mice were significantly lower than WT mice even under the phenobarbital-induced conditions, which differed from physiologic conditions. These results suggest that although P450 activities were lower in $\mathrm{Nrf} 2^{-1-}$ mice under both physiologic and induced conditions, the contributing P450 species were different. Phenobarbital substantially induced Cyp2b10 compared with other $\mathrm{P} 450$ genes, and Cyp2b10 levels were decreased in $\mathrm{Nrf}^{-/-}$mice compared with WT mice. Phenobarbital-induced sleeping time was previously reported to be associated with CYP2B activity (Sirisangtragul and Sripanidkulchai, 2013). Taken together, the phenobarbitalshortened pentobarbital efficiency and its inhibition in $\mathrm{Nrf} 2^{-/-}$mice may depend on CYP2B activity.

In this study, $\mathrm{Nrf} 2^{-1-}$ mice reliably showed prolonged pentobarbitalinduced sleeping time; however, the mechanism underlying the wide range of individual differences in the sleeping time in $\mathrm{Nrf} 2^{-1-}$ mice under physiologic conditions remains unclear. It has been reported that a congenital intrahepatic shunt is present at a constant rate in Nrf2disrupted C57BL/6J mice (Skoko et al., 2014). This intrahepatic shunt would be expected to change blood flow, which may result in the individual differences in sleeping time of $\mathrm{Nrf} 2^{-1-}$ mice. However, individual differences in blood concentration of pentobarbital were not 
observed in $\mathrm{Nrf} 2^{-1-}$ mice. Furthermore, individual differences in sleeping time were also not observed in the phenobarbitaladministered $\mathrm{Nrf}^{-1-}$ mice, which may contribute in part to the wide range of individual differences in the sleeping time in $\mathrm{Nrf} 2^{-/-}$mice under physiologic conditions.

Expression of P450 genes is influenced by various physiologic changes and exogenous factors. Several pathophysiological conditions, such as infection and inflammation, have been shown to suppress expression of several P450 isoforms (Morgan, 1997). Although this is well studied in terms of regulation of inflammatory cytokines such as interleukin- $1 \beta$, interleukin-6, and tumor necrosis factor $\alpha$ (Ashino et al., 2004), little is known about the underlying mechanism of P450 downregulation, which could involve ROS generation by inflammatory cytokine signaling. Indeed, CYP1A1 and CYP2E1 gene expression levels have been shown to be reduced by ROS production in response to $\mathrm{H}_{2} \mathrm{O}_{2}$ treatment (Morel and Barouki, 1998; Morel et al., 2000), suggesting that other $\mathrm{P} 450$ species are also repressed by ROS. Furthermore, this study showed that the affinity of metabolic enzymes to pentobarbital was not altered by Nrf2 depletion. Taken together, an increase in ROS by Nrf2 depletion may negatively regulate P450 gene expression, thereby suppressing their activities without affecting the P450 structural conformation.

Transcriptional activation of P450s has been mainly investigated in the context of several xenobiotic-activated receptors, such as aryl hydrocarbon receptor, constitutive androstane receptor (CAR), and pregnane X receptor (Rushmore and Kong, 2002), which act as specific transcription factors. Previous investigations have demonstrated that $\mathrm{Nrf} 2$ functions as a transcription factor for the Cyp2a5 gene by directly binding to the promoter region (Abu-Bakar et al., 2007; Wu et al., 2012; Ashino et al., 2014). This study also showed that Cyp2a5 gene expression was regulated by $\mathrm{Nrf} 2$, indicating Cyp2a5 as the first phase I xenobiotic-metabolizing gene identified under the regulation of the $\mathrm{Nrf} 2$ pathway with a potential role in the adaptive response to cellular stress. Although the Nrf2-binding site ARE/StRE has not been identified on Cyp1a2, Cyp2c29, and Cyp2e1 genes, their expression levels were downregulated in Nrf2 $2^{-1-}$ mice. Thus, Nrf2 depletion-suppressed ROS elimination is likely involved in this regulation.

Phenobarbital is the prototype of a large group of structurally diverse xenobiotic chemicals that induce the subset of the $\mathrm{P} 450$ genes such as CYP2B, CYP2C, and CYP3A subfamilies, with CYP2B genes being the most effectively induced (Honkakoski et al., 1998). Although constitutive expression of the CYP2B subfamily is very low, their expression levels are dramatically increased by specific inducers. Phenobarbital has been demonstrated to transactivate the CYP2B gene through nuclear translocation of CAR, in which it forms a heterodimer with the retinoid $\mathrm{X}$ receptor and activates the phenobarbital responsive element module (Honkakoski et al., 1998; Kawamoto et al., 1999; Moore et al., 2000). It has been reported that Nrf2 activators, such as trans-stilbeneoxide and oltipraz, also induce Cyp2b10 gene expression and activate the CYP2B6 promoter (Slitt et al., 2006; Merrell et al., 2008). However, ARE/StRE have not been detected in its promoter regions, indicating no direct transcriptional target of $\mathrm{Nrf} 2$. Induction of Cyp2b10 by Nrf2 activators is inhibited in $\mathrm{CAR}^{-1-}$ mice, but not $\mathrm{Nrf}^{-1-}$ mice (Merrell et al., 2008). Although it remains unclear how Nrf2 activators activates CAR, these reports suggest that several Nrf2 activators can activate CAR, followed by Cyp2b10 induction. Thus, suppressed induction of Cyp2b10 in $\mathrm{Nrf} 2^{-l-}$ mice may be involved in the increase in ROS levels due to Nrf2 depletion.

This study also showed that Nrf2 depletion downregulated ferritin gene expression, a Nrf2 target gene. The iron storage protein, ferritin, has been postulated to act as an intermediate for heme synthesis (Richardson and Ponka, 1997). The liver is one of the body compartments with the highest rate of heme synthesis. More than $50 \%$ of heme synthesized in the liver is used to synthesize P450s (Vinchi et al., 2014). Therefore, it is speculated that ferritin expression in livers may influence P450 maturation and activation through heme incorporation. It has been reported that the CYP2A6 activity is related to serum ferritin levels, which are correlated to intracellular ferritin levels (Satarug et al., 2004). These results suggest that Nrf2 plays an important role in the variance of pharmacokinetics of administered drugs, at least, in part, by regulating ferritin expression that may contribute to a delay in pentobarbital metabolism in $\mathrm{Nrf}^{-1-}$ mice.

In conclusion, this study revealed a role of $\mathrm{Nrf} 2$ as a maintenance factor of drug metabolizing enzyme $\mathrm{P} 450$ s through transcriptional modulation of gene expression. Although further studies are required to elucidate the underlying mechanisms of P450 downregulation in biologic defense, we suggest that the Nrf2 system inhibits an increase in ROS by regulating the metabolic cycle of P450s, thereby maintaining homeostasis against oxidative damage. Therefore, Nrf2 dysfunction or ROS that exceed a threshold level of the eliminating ability of the Nrf2 system may reduce P450 activity. Our findings also highlight the Nrf2 system as a predictive factor for the duration and intensity of intended drug efficacies.

\section{Acknowledgments}

The authors thank K. Higeno for his technical assistance. The authors would like to thank Editage (www.editage.com) for the English language review.

\section{Authorship Contributions}

Participated in research design: Ashino.

Conducted experiments: Ashino.

Contributed new reagents or analytic tools: Yamamoto.

Performed data analysis: Ashino, Numazawa.

Wrote or contributed to the writing of the manuscript: Ashino.

\section{References}

Abu-Bakar A, Lämsä V, Arpiainen S, Moore MR, Lang MA, and Hakkola J (2007) Regulation of CYP2A5 gene by the transcription factor nuclear factor (erythroid-derived 2)-like 2. Drug Metab Dispos 35:787-794.

Aitken AE, Richardson TA, and Morgan ET (2006) Regulation of drug-metabolizing enzymes and transporters in inflammation. Annu Rev Pharmacol Toxicol 46:123-149.

Ashino T, Arima Y, Shioda S, Iwakura Y, Numazawa S, and Yoshida T (2007) Effect of interleukin-6 neutralization on CYP3A11 and metallothionein-1/2 expressions in arthritic mouse liver. Eur J Pharmacol 558:199-207.

Ashino T, Oguro T, Shioda S, Horai R, Asano M, Sekikawa K, Iwakura Y, Numazawa S, and Yoshida T (2004) Involvement of interleukin-6 and tumor necrosis factor alpha in CYP3A11 and 2C29 down-regulation by Bacillus Calmette-Guerin and lipopolysaccharide in mouse liver. Drug Metab Dispos 32:707-714.

Ashino T, Ohkubo-Morita H, Yamamoto M, Yoshida T, and Numazawa S (2014) Possible involvement of nuclear factor erythroid 2-related factor 2 in the gene expression of Cyp2b10 and Cyp2a5. Redox Biol 2:284-288.

Ashino T, Sugiuchi J, Uehara J, Naito-Yamamoto Y, Kenmotsu S, Iwakura Y, Shioda S, Numazawa S, and Yoshida T (2011) Auranofin protects against cocaine-induced hepatic injury through induction of heme oxygenase-1. J Toxicol Sci 36:635-643.

Aubert J, Begriche K, Knockaert L, Robin MA, and Fromenty B (2011) Increased expression of cytochrome P450 2E1 in nonalcoholic fatty liver disease: mechanisms and pathophysiological role. Clin Res Hepatol Gastroenterol 35:630-637.

Barouki R and Morel Y (2001) Repression of cytochrome P450 1A1 gene expression by oxidative stress: mechanisms and biological implications. Biochem Pharmacol 61:511-516.

Eid AA, Gorin Y, Fagg BM, Maalouf R, Barnes JL, Block K, and Abboud HE (2009) Mechanisms of podocyte injury in diabetes: role of cytochrome P450 and NADPH oxidases. Diabetes $\mathbf{5 8}$ $1201-1211$.

Frye RF, Schneider VM, Frye CS, and Feldman AM (2002) Plasma levels of TNF-alpha and IL-6 are inversely related to cytochrome P450-dependent drug metabolism in patients with congestive heart failure. J Card Fail 8:315-319.

Gonzalez FJ (1988) The molecular biology of cytochrome P450s. Pharmacol Rev 40:243-288.

Griffiths HR, Gao D, and Pararasa C (2017) Redox regulation in metabolic programming and inflammation. Redox Biol 12:50-57.

Haas CE, Kaufman DC, Jones CE, Burstein AH, and Reiss W (2003) Cytochrome P450 3A4 activity after surgical stress. Crit Care Med 31:1338-1346.

Hakkola J, Bernasconi C, Coecke S, Richert L, Andersson TB, and Pelkonen O (2018) Cytochrome $\mathrm{P} 450$ induction and xeno-sensing receptors pregnane $\mathrm{X}$ receptor, constitutive androstane receptor, aryl hydrocarbon receptor and peroxisome proliferator-activated receptor $\alpha$ at the crossroads of toxicokinetics and toxicodynamics. Basic Clin Pharmacol Toxicol 123 (Suppl 5):42-50.

Honkakoski P, Zelko I, Sueyoshi T, and Negishi M (1998) The nuclear orphan receptor CARretinoid $\mathrm{X}$ receptor heterodimer activates the phenobarbital-responsive enhancer module of the CYP2B gene. Mol Cell Biol 18:5652-5658. 
Isin EM and Guengerich FP (2007) Complex reactions catalyzed by cytochrome P450 enzymes. Biochim Biophys Acta 1770:314-329.

Itoh K, Chiba T, Takahashi S, Ishii T, Igarashi K, Katoh Y, Oyake T, Hayashi N, Satoh K, Hatayama I, et al. (1997) An Nrf2/small Maf heterodimer mediates the induction of phase II detoxifying enzyme genes through antioxidant response elements. Biochem Biophys Res Commun 236:313-322.

Kawamoto T, Sueyoshi T, Zelko I, Moore R, Washburn K, and Negishi M (1999) Phenobarbitalresponsive nuclear translocation of the receptor CAR in induction of the CYP2B gene. Mol Cell Biol 19:6318-6322.

Kelner M and Bailey DN (1983) Reversed-phase liquid-chromatographic simultaneous analysis for thiopental and pentobarbital in serum. Clin Chem 29:1097-1100.

Kovács T, Déri M, Fülöp A, Pálházy T, Háfra E, Sirok D, Kiss AF, Lotz G, Szijártó A, and Monostory K (2018) Isoform-dependent changes in cytochrome P450-mediated drug metabolism after portal vein ligation in the rat. Eur Surg Res 59:301-319.

Mayo PR, Skeith K, Russell AS, and Jamali F (2000) Decreased dromotropic response to verapamil despite pronounced increased drug concentration in rheumatoid arthritis. $\mathrm{Br} \mathrm{J}$ Clin Pharmacol 50:605-613.

Merrell MD, Jackson JP, Augustine LM, Fisher CD, Slitt AL, Maher JM, Huang W, Moore DD Zhang Y, Klaassen CD, et al. (2008) The Nrf2 activator oltipraz also activates the constitutive androstane receptor. Drug Metab Dispos 36:1716-1721.

Moore LB, Parks DJ, Jones SA, Bledsoe RK, Consler TG, Stimmel JB, Goodwin B, Liddle C, Blanchard SG, Willson TM, et al. (2000) Orphan nuclear receptors constitutive androstane receptor and pregnane $\mathrm{X}$ receptor share xenobiotic and steroid ligands. J Biol Chem $\mathbf{2 7 5}$ 15122-15127.

Morel Y and Barouki R (1998) Down-regulation of cytochrome P450 1A1 gene promoter by oxidative stress. Critical contribution of nuclear factor 1. J Biol Chem 273:26969-26976.

Morel Y, de Waziers I, and Barouki R (2000) A repressive cross-regulation between catalytic and promoter activities of the CYP1A1 and CYP2E1 genes: role of $\mathrm{H}(2) \mathrm{O}(2)$. Mol Pharmacol 57: $1158-1164$.

Morgan ET (1997) Regulation of cytochromes P450 during inflammation and infection. Drug Metab Rev 29:1129-1188

Oguro T, Takahashi Y, Ashino T, Takaki A, Shioda S, Horai R, Asano M, Sekikawa K, Iwakura Y, and Yoshida T (2002) Involvement of tumor necrosis factor alpha, rather than interleukin-1alph beta or nitric oxides in the heme oxygenase-1 gene expression by lipopolysaccharide in the mouse liver. FEBS Lett 516:63-66.

Omura T and Sato R (1964) The carbon monoxide-binding pigment of liver microsomes. I Evidence for its hemoprotein nature. J Biol Chem 239:2370-2378.

Richardson DR and Ponka P (1997) The molecular mechanisms of the metabolism and transport of iron in normal and neoplastic cells. Biochim Biophys Acta 1331:1-40.

Roberts CK and Sindhu KK (2009) Oxidative stress and metabolic syndrome. Life Sci 84:705-712.
Rushmore TH and Kong AN (2002) Pharmacogenomics, regulation and signaling pathways of phase I and II drug metabolizing enzymes. Curr Drug Metab 3:481-490.

Sahan-Firat S, Jennings BL, Yaghini FA, Song CY, Estes AM, Fang XR, Farjana N, Khan AI, and Malik KU (2010) 2,3',4,5'-Tetramethoxystilbene prevents deoxycorticosterone-salt-induced hypertension: contribution of cytochrome P-450 1B1. Am J Physiol Heart Circ Physiol 299: H1891-H1901.

Satarug S, Ujjin P, Vanavanitkun Y, Nishijo M, Baker JR, and Moore MR (2004) Effects of cigarette smoking and exposure to cadmium and lead on phenotypic variability of hepatic CYP2A6 and renal function biomarkers in men. Toxicology 204:161-173.

Sirisangtragul W and Sripanidkulchai B (2013) Moduratory effect of Thai traditional medicine (Yahom Tultavai) on hepatic cytochrome P450 enzymes and pentobarbital-induced sleeping in mice. Afr J Tradit Complement Altern Med 10:128-136.

Skoko JJ, Wakabayashi N, Noda K, Kimura S, Tobita K, Shigemura N, Tsujita T, Yamamoto M, and Kensler TW (2014) Loss of Nrf2 in mice evokes a congenital intrahepatic shunt that alters hepatic oxygen and protein expression gradients and toxicity. Toxicol Sci 141:112-119.

Slitt AL, Cherrington NJ, Dieter MZ, Aleksunes LM, Scheffer GL, Huang W, Moore DD, and Klaassen CD (2006) trans-Stilbene oxide induces expression of genes involved in metabolism and transport in mouse liver via CAR and Nrf2 transcription factors. Mol Pharmacol 69: $1554-1563$.

Tsuji R, Isobe N, Kurita Y, Hanai K, Yabusaki Y, and Kawasaki H (1996) Species difference in the inhibition of pentobarbital metabolism by empenthrin. Environ Toxicol Pharmacol 2:331-337.

Vinchi F, Ingoglia G, Chiabrando D, Mercurio S, Turco E, Silengo L, Altruda F, and Tolosano E (2014) Heme exporter FLVCR1a regulates heme synthesis and degradation and controls activity of cytochromes P450. Gastroenterology 146:1325-1338.

Wang H and Negishi M (2003) Transcriptional regulation of cytochrome p450 2B genes by nuclear receptors. Curr Drug Metab 4:515-525.

Wu KC, Cui JY, and Klaassen CD (2012) Effect of graded Nrf2 activation on phase-I and -II drug metabolizing enzymes and transporters in mouse liver. PLoS One 7:e39006.

Yamamoto M, Kensler TW, and Motohashi H (2018) The KEAP1-NRF2 system: a thiol-based sensor-effector apparatus for maintaining redox homeostasis. Physiol Rev 98:1169-1203.

Zangar RC, Davydov DR, and Verma S (2004) Mechanisms that regulate production of reactive oxygen species by cytochrome P450. Toxicol Appl Pharmacol 199:316-331.

Address correspondence to: Dr. Takashi Ashino, Division of Toxicology, Department of Pharmacology, Toxicology, and Therapeutics, Showa University School of Pharmacy, 1-5-8 Hatanodai, Shinagawa-ku, Tokyo 142-8555, Japan. E-mail: ashino@pharm.showa-u.ac.jp 